small proportion (4\%) indicated they had never had a Pap test. In multivariate models, having a Pap test in the past year was negatively associated with income $>3500$ pesos/month, more years in the sex trade and having regular clients. Marginal positive associations remained with older age, reporting condom use less than half the time with non-regular clients and having any children.

Discussion Prevalence of pap tests in the past year was higher than expected and may be attributed to recent efforts by the Tijuana Municipal Health Services to increase outreach to FSWs in these areas. However, since initiating sex work, only half reported the recommended yearly Pap testing which is concerning given the increased risk for HPV infection and cervical cancer among FSW. Sexual health education, including where access services, is needed to encourage regular cancer screening among this high risk population, especially among younger women and women who have been working in the sex trade for longer durations.

\section{P5-S6.32 USING A CONTINGENT VALUATION METHOD TO UNDERSTAND CONSUMER PREFERENCES FOR CARE OF ADOLESCENTS WITH PELVIC INFLAMMATORY DISEASE (PID)}

doi:10.1136/sextrans-2011-050108.588

${ }^{1} \mathrm{M}$ Trent, ${ }^{2} \mathrm{O}$ Oian, ${ }^{3} \mathrm{~K}$ Frick, ${ }^{3} \mathrm{C}$ Thompson, ${ }^{4} \mathrm{~A}$ Butz, ${ }^{4} \mathrm{~J}$ Ellen, ${ }^{4} \mathrm{H}$ Lehmann. ${ }^{1} \mathrm{~J}$ ohns Hopkins University School of Medicine, Baltimore, USA; ${ }^{2}$ University of Maryland, Towson, USA; ${ }^{3}$ Johns Hopkins Bloomberg School of Public Health, Baltimore, USA; ${ }^{4}$ Johns Hopkins School of Medicine, Baltimore, USA

Objective Adolescent girls diagnosed with PID are at higher risk for subsequent sexually transmitted infection (STI), pregnancy, and long-term pelvic pain. Although the 72 -h post-PID evaluation provides an opportunity for risk reduction counselling, few adolescents adhere. Use of public health nurses (PHN) for clinical followup may meet the needs of this vulnerable population. The objective of this study is to estimate consumers' willingness-to-pay (WTP) for follow-up PID services by physicians and PHNs, differences by consumer type, and the differences in health-provider predicted consumer WTP values and actual consumer WTP values.

Methods A contingent valuation method was used to collect WTP data regarding co-payments to physicians or nurses for clinical service delivery from the consumers of adolescent PID services (parents $(n=121)$ and adolescents $(n=134))$ and a national sample of health providers $(n=102)$. Consumers were recruited from an academic paediatric practice and school-based health clinics in a large urban community with high STI prevalence. Participants completed a web-based survey with data uploaded to a secure server after obtaining online consent. Data were analysed using linear regression analyses.

Results The mean WTP for physician services was \$16 (SD \$16.9) for clinicians, $\$ 81.9$ (\$34.0) for parents, and $\$ 72$ (SD \$39.1) for adolescents. The mean WTP for PHN services was \$13.6 (SD \$17.4) for physicians, \$62.4 (SD \$44.1) for parents, and \$49.7 (SD \$44) for adolescents. Using physician estimates for WTP as the reference group, adolescents were willing to pay $\$ 56$ more (95\% CI 48.6 to 63.4 ) for physician care and parents were willing to pay $\$ 66$ more (95\% CI 59.0 to 72.8 ) than physician's predicted controlling for informant employment status. Adolescents were willing to pay $\$ 36$ more (95\% CI 48.6 to 63.4 ) for community-based nursing care and parents were willing to pay $\$ 48$ more ( $95 \%$ CI 59.0 to 72.8 ) than physician's predicted. Consumers' (adolescents' \& parents') WTP for physician services were on average $\$ 18.50$ higher than PHN services $(\mathrm{p}=0.01)$.

Conclusion While adolescents and parents prefer physician followup for PID based on WTP, they are amenable to PHN follow-up visits. Our data suggest that health providers underestimate the value the consumers place on clinical service for PID. Given poor adherence to office-based follow-up and consumer interest in PHN visits, additional research evaluating the effectiveness of PHN visits for PID is warranted.

\section{P5-S6.33 HIV/AIDS IMPACT MITIGATION IN RESOURCE-POOR SETTINGS: SCALING-UP A MULTI-SECTORAL RESPONSE}

doi:10.1136/sextrans-2011-050108.589

${ }^{1}$ A Mwendwa, ${ }^{2} \mathrm{~N}$ Kyalo, ${ }^{3}$ W Kihara, ${ }^{4} \mathrm{~J}$ Mburu, ${ }^{5} \mathrm{M}$ Mutua. ${ }^{1}$ Moi University Hospital Teaching and Referral Centre, Eldoret, Kenya; ${ }^{2}$ University of Nairobi, Nairobi, Kenya; ${ }^{3}$ Kenyatta National Hospital, Nairobi, Kenya; ${ }^{4}$ Redeemed Gospel Church Development Programme, Nairobi, Kenya; ${ }^{5}$ Centre for Integrated Community Development and Outreach, CICDOT, Nairobi, Kenya

Background/issues The HIV and AIDS epidemic constitutes not only the worst scourge and onslaught with which peoples in resourcelimited places must contend; It also occurs in a context in which the effects of the epidemic and key issues which they engender are juxtaposed with a multiplicity of societal problems. Many high HIV/AIDS prevalence countries apparently remain unconvinced of the longer term impact of this epidemic, and have not yet developed strategies to cope with the obvious and incontrovertible impact of HIV and AIDS. Impact mitigation of HIV and AIDS is not high on the agenda of the many organisations which are involved in mounting a response to this epidemic. These organisations have assumed HIV/AIDS to be a public health issue and failed in many cases to recognise it for the socio-economic development challenge it more properly represents. Empirical research findings and Observations/In resource poor settings, greed and avarice of the political leadership overwhelms their responsibilities take urgent action to mitigate HIV/AIDS impact. There are small islands of dazzling abundance which exist side by side with a cheerless ocean of absolute poverty, dehumanising HIV/AIDS high prevalence rate and wide-scale social exclusion. Africa's famed traditional extended family system, exemplary at absorbing members under stress, is confronted with HIV and AIDS impact on every constituent part of its network and may reach breaking point without some assistance. This strain may have the effect of not only accelerating the reversal of development gains of the last decade but of fragmenting the very societal structure that has so far sustained marginal societies.

Conclusions/policy implications There's the pressing moral, social, political and economic imperative to scale up the impact mitigation of HIV/AIDS among residents of poor areas. The immediate focus of managing the crisis of HIV and AIDS in resource-limited settings will impact every aspect of multi-sectoral, systemic functioning, response. The advent of a vastly improved prognosis of new classes of ARV drugs and their use in combination can dramatically improve rates of mortality and morbidity, prolonged lives, improved quality of life, revitalised communities and transformed perceptions of HIV/AIDS from a plague to a manageable, chronic illness in resource poor settings.

\section{P5-S6.34 PROVIDER CHARACTERISTICS ASSOCIATED WITH GONORRHOEA TREATMENT ERRORS, MASSACHUSETTS, 2010}

doi:10.1136/sextrans-2011-050108.590

${ }^{1} \mathrm{~J}$ Swails, ${ }^{2} \mathrm{~L}$ Smock, ${ }^{2} \mathrm{Y}$ Tang, ${ }^{2} \mathrm{~K}$ Hsu. ${ }^{1}$ Brigham and Women's Hospital, Boston, USA; ${ }^{2}$ Massachusetts Department of Public Health USA

Background Massachusetts experienced a $29 \%$ increase in gonorrhoea cases during 2010. In $4 \%$ of cases where treatment was known, treatment was inconsistent with public health guidelines. We examined healthcare provider characteristics associated with gonorrhoea treatment errors to help target future educational outreach strategies. 